\title{
Electro-optical circuits based on light-sensing ambipolar organic field-effect transistors
}

\author{
Thomas D. Anthopoulos ${ }^{\text {a) }}$ \\ Department of Physics, Blackett Laboratory, Imperial College London, London SW7 2BW, United Kingdom
}

(Received 18 June 2007; accepted 12 August 2007; published online 13 September 2007)

The author reports on light-sensing ambipolar organic field-effect transistors (LS-OFETs) based on blends of[6,6]-phenyl- $\mathrm{C}_{61}$-butyric acid methyl ester and poly [2-methoxy-5$\left(3^{\prime}, 7^{\prime}\right.$-dimethyloctyloxy)]-p-phenylene vinylene. By carefully tuning the ambipolar transport character of the LS-OFETs, their photoresponsivity can be controlled and optimized. By combining LS-OFETs with ordinary unipolar organic transistors, fabrication of electro-optical switches and logic circuits, such as NOT and OR gates, is demonstrated. This is the only report of organic electro-optical circuits in which signal processing involves the use of both optical and electrical input signals. Such circuits could one day be explored in optical sensor array applications.

(C) 2007 American Institute of Physics. [DOI: 10.1063/1.2778754]

Over the past twenty years progress in the area of organic field-effect transistors (OFETs) and integrated circuits has been driven mainly by the development of organic semiconductors rather than advancements in device and circuit design concepts. In particular, use of OFETs has been restricted in applications where the transistors are used purely as unifunctional devices (e.g., current switches), notably in pixel engines for active-matrix flexible displays ${ }^{1}$ and organic integrated circuits. ${ }^{2,3}$ Recently, however, organic transistors with additional functionalities, i.e., bifunctional OFETs, have been designed and demonstrated with most notable examples the light-emitting ${ }^{4-6}$ and light-sensing ${ }^{7-9}$ (LS-OFETs) transistors. These latter demonstrations are of particular significance since, in principle, design and fabrication of a different breed of organic electro-optical circuits in which the electrical and optical functionalities of these bifunctional transistors are combined, can be envisioned. To this end, of primary importance is the development and demonstration of highperformance bifunctional organic transistors, and in particular LS-OFETs, and their use in technological applications.

The present work reports on electro-optical circuits based on ambipolar LS-OFETs and ordinary unipolar OFETs. The former are realized using blends of $[6$ $6]$-phenyl- $\mathrm{C}_{61}$-butyric acid methyl ester $([60] \mathrm{PCBM})$ and poly [2-methoxy-5-(3', 7'-dimethyloctyloxy- $p$-phenylene vinylene $\left(\mathrm{OC}_{1} \mathrm{C}_{10} \mathrm{PPV}\right)$, while the latter using pristine [60]PCBM. By integrating the LS-OFETs with $n$-channel OFETs, we are able to demonstrate various optoelectronic circuits including switches and logic gates such as NOT and OR. A unique characteristic of these gates is that the input signal(s) can be designed to be either purely optical or a combination of electrical and optical. An additional advantage of this technology, and unlike any other organic-based photodetector, is that LS-OFETs can be integrated with the driving electronics (i.e., unipolar OFETs) in parallel and side by side employing the same number of processing steps, hence, eliminating the need of additional fabrication costs. ${ }^{10}$

Field-effect transistors were made using heavily doped $\mathrm{Si}^{++}$wafers as the global gate electrode with a $200 \mathrm{~nm}$ thermally oxidized $\mathrm{SiO}_{2}$ layer as the gate dielectric. Using conventional photolithography, gold source and drain electrodes

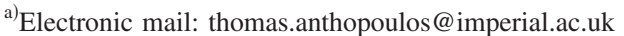

were defined in a bottom-contact configuration with channel length $(L)$ and width $(W)$ in the range of $1-60 \mu \mathrm{m}$ and 1-10 mm, respectively. A $10 \mathrm{~nm}$ layer of titanium was used acting as an adhesion layer for the gold on $\mathrm{SiO}_{2}$. The $\mathrm{SiO}_{2}$ layer was treated with the primer hexamethyldisilazane prior to semiconductor deposition, in order to passivate its surface. Films were spin cast from a $10 \mathrm{mg} / \mathrm{ml}$ solution of [60]PCBM: $\mathrm{OC}_{1} \mathrm{C}_{10} \mathrm{PPV}(15: 1 \mathrm{wt} \%)$ in chlorobenzene at room temperature. Scientific grade $99.5 \%$ [60]PCBM was purchased from Solenne B.V., and $\mathrm{OC}_{1} \mathrm{C}_{10} \mathrm{PPV}$ from Sigma Aldrich, Inc. Electrical characterization was performed using an HP 4156C semiconductor parameter analyzer. The dynamic characteristics were measured using a current amplifier and a $1 \mathrm{M} \Omega$ input impedance oscilloscope. As the light source a blue (469 nm) InGaN light emitting diode (LED) was employed in close proximity to the device. All measurements were performed at room temperature in high vacuum $\left(10^{-5}\right.$ mbar) or under $\mathrm{N}_{2}$ at atmospheric pressure.

Figure 1(a) displays the ambipolar transfer characteristics of a [60]PCBM: $\mathrm{OC}_{1} \mathrm{C}_{10} \mathrm{PPV}(15: 1 \mathrm{wt} \%)$ based transistor $L=2 \mu \mathrm{m}, W=20 \mathrm{~mm}$ ) measured in the dark (solid lines) and under constant illumination (circles), using a blue LED, at different drain voltages $\left(V_{D}\right)$. The ambipolar carrier mobilities were calculated from the transfer curves obtained in saturation in the dark, yielding values on the order $10^{-4}-10^{-3} \mathrm{~cm}^{2} / \mathrm{V}$ s for holes and electrons. LS-OFETs based on blends containing lower concentrations of [60]PCBM (e.g., 4:1 wt \% [60]PCBM: $\mathrm{OC}_{1} \mathrm{C}_{10} \mathrm{PPV}$ ) were found to exhibit lower electron mobility and photosensitivity $(P)$. The threshold voltages, calculated from the linear parts of the corresponding $I_{D}^{1 / 2}$ vs $V_{G}$ plots, were $\sim 20$ and $\sim 5 \mathrm{~V}$ for the $n$ and $p$ channels, respectively. Devices characterized in the dark and under high vacuum (solid lines) show no significant hysteresis. The pinch-off current, measured at low $V_{D}$ in the dark $\left(I_{\mathrm{OFF}}\right)$, is very low and at the edge of our detection limit. When the device is illuminated with blue light $(469 \mathrm{~nm})$, however, the pinch-off current $\left[I_{\mathrm{OFF}(\mathrm{LED})}\right]$ increases by several orders of magnitude (circles). Using the transfer curves measured in the dark and under illumination, the photosensitivity of the LS-OFET was calculated as the ratio of $I_{\mathrm{OFF}(\mathrm{LED})}$ to $I_{\mathrm{OFF}}$ yielding a maximum value on the order of $P \sim 10^{4}$. The latter depends both on $V_{D}$ and the intensity of 

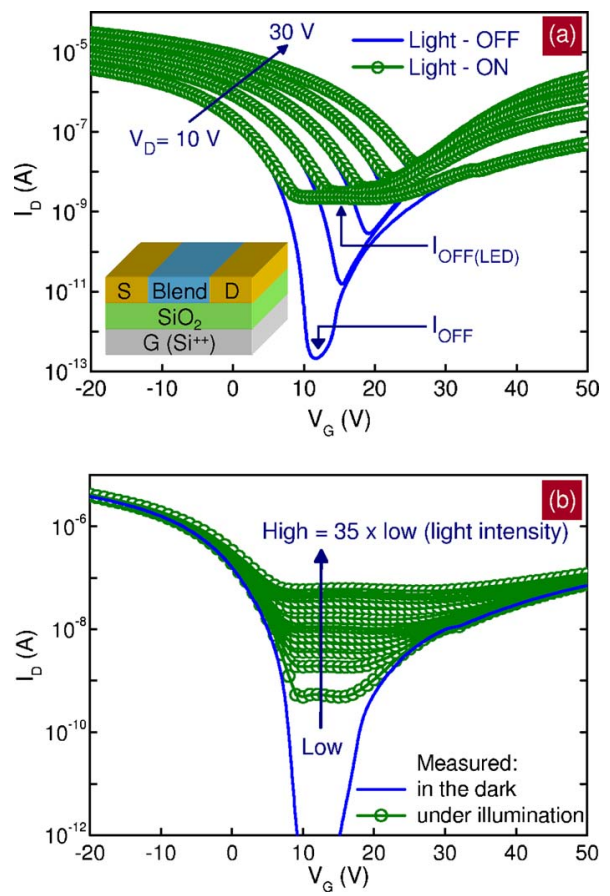

FIG. 1. (Color online) (a) Transfer curves of an ambipolar LS-OFET $(L=2 \mu \mathrm{m}, W=10 \mathrm{~mm})$ based on [60]PCBM: $\mathrm{OC}_{1} \mathrm{C}_{10} \mathrm{PPV}$ (15:1 wt \%) blend. Measurements were performed in the dark (solid line) and under constant illumination (circles) using an inorganic blue (469 nm) InGaN LED. Inset shows the bottom-gate, bottom-contact transistor architecture employed. b) Transfer characteristics of the same LS-OFET measured in the dark (solid line) and under illumination at different light intensities (circles) at $V_{D}=10 \mathrm{~V}$.

the incident radiation. The $P$ dependence on $V_{D}$ is due to the increase of $I_{\mathrm{OFF}}$ with increasing $V_{D}$ [Fig. 1(a), solid lines], a common characteristic of ambipolar organic transistors. ${ }^{11}$ The strong and nearly linear dependence of $I_{\mathrm{OFF}}$ on the incident light intensity, on the other hand [Fig. 1(b)], is attributed to the increased number of photogenerated carriers at higher intensities. The exact physical mechanism responsible for this effect is not yet fully understood and is beyond the scope of this work.

The dynamic response of the LS-OFETs, was measured employing the circuit shown in Fig. 2(a). Here, CA is a low current amplifier and the light source is a blue LED $(469 \mathrm{~nm})$. A typical set of measurements, obtained at $V_{D D}$ $=10 \mathrm{~V}$ and $V_{G}=15 \mathrm{~V}$, is shown in Fig. 2(b). The red trace shows the light intensity (as detected using a Si photodiode) incident on the surface of the LS-OFET. The blue trace shows the voltage at the output of the CA $\left(\propto I_{D}\right)$. Interestingly and unlike previously published work, ${ }^{7-9}$ the ambipolar LS-OFET is fast with switching times $<1 \mathrm{~ms}$. Analysis of the rise $\left(t_{R}\right)$ and fall $\left(t_{F}\right)$ times shown in Fig. 2(c) yields a minimum total response time $t_{T}=\left(t_{R}+t_{F}\right)$ of $\sim 65 \mu \mathrm{s}$, which corresponds to an operating frequency $(f)$ of $\sim 15 \mathrm{kHz}$ calculated using $f=1 / t_{T}$.

To demonstrate the potential of the technology in practical circuit applications, we first realize a simple electrooptical NOT gate [Fig. 3(a)]. The circuit is comprized of two organic transistors, one LS-OFET (T2) and one ordinary $n$ channel [60]PCBM-based OFET (T1). ${ }^{12,13}$ In this particular case $\mathrm{T} 1$ and $\mathrm{T} 2$ were integrated in a complementary layout, however, different circuit designs could be adopted. A representative set of results obtained from this circuit is shown in Fig. 3(b). When the optical input signal $A_{\text {IN }}$ (red trace) is Downloaded 25 Apr 2008 to 155.198.4.89. Redistribution subject

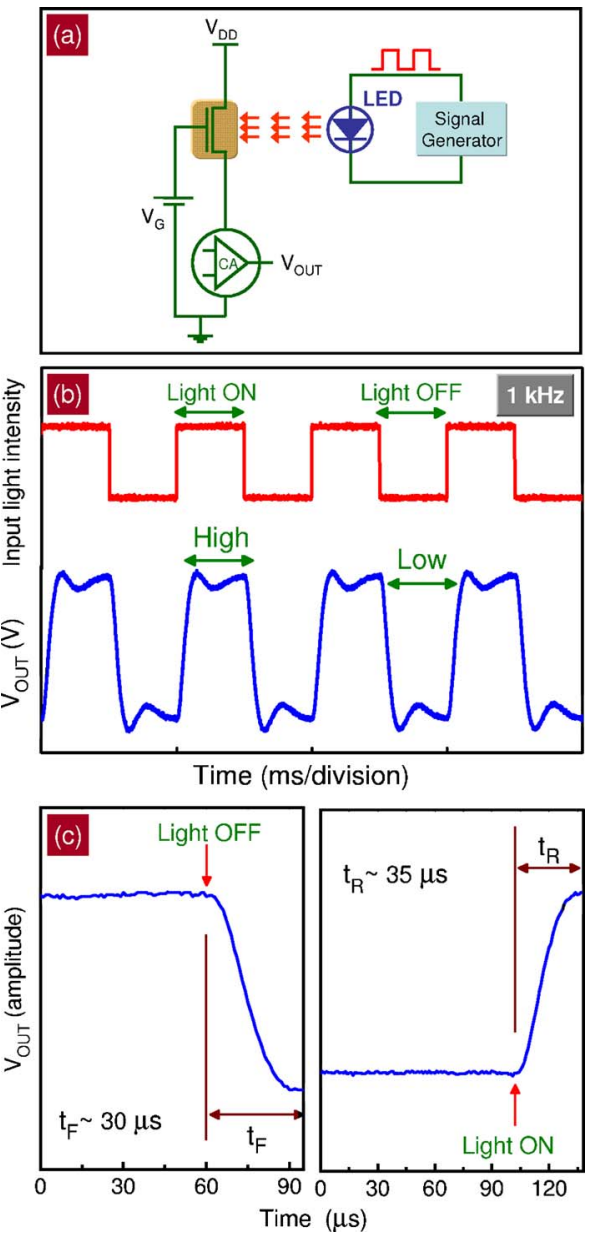

FIG. 2. (Color online) (a) Circuitry of the experimental setup employed for the dynamic characterization of LS-OFETs. (b) Evolution of the channel current (blue trace) as a function of a pulsed $(1 \mathrm{kHz})$ light input signal (red trace) vs time. (c) Fall $\left(t_{F}\right)$ and rise $\left(t_{R}\right)$ times of $V_{\text {OUT }}$ vs time.

high, i.e., light is on, the impedance of $\mathrm{T} 2\left(R_{T 2}\right)$ reduces to a value much lower than that of T1 $\left(R_{T 1}\right)$ due to the photoinduced charges in the channel. As a result, the voltage drop across $\mathrm{T} 2$ is negligible, as compared to the voltage drop across $\mathrm{T} 1$, leading to a low voltage output so $V_{\mathrm{OUT}}=$ low (blue trace). If the optical input signal $A_{\mathrm{IN}}$ (red trace) is switched to the low state, i.e., light off, the voltage drop across $\mathrm{T} 2$ is much larger than that across $\mathrm{T} 1$, since $R_{T 2}$ $\gg R_{T 1},{ }^{14}$ pulling $V_{\text {OUT }}$ at a higher voltage level so $V_{\text {OUT }}$ $=$ high. In both cases, inversion of the optical input signal is demonstrated for either binary state of $A_{\mathrm{IN}}$. These results are in agreement with the truth table of a standard NOT gate shown in the inset of Fig. 3(a).

Using the same design principles, other electro-optical organic circuits incorporating a combination of electrical and optical input signals can, in principle, be designed and fabricated. A representative example is the electro-optical OR gate shown in Fig. 4(a). For simplicity, a single LS-OFET similar to the one used in Fig. 3 is employed. The circuit has two input signals, one electrical $\left(A_{\mathrm{IN}}\right)$ and one optical $\left(B_{\mathrm{IN}}\right)$. When either of the signals is high (i.e., $A_{\mathrm{IN}}=$ high or $B_{\mathrm{IN}}$ $=$ light on $)$, the output signal $\left(I_{\text {OUT }}\right)$, i.e., the current flowing through the transistor channel, increases and reaches a steady state that corresponds to the high output binary state. Only when both inputs are in their low state (i.e., $A_{\mathrm{IN}}=$ Low and $B_{\mathrm{IN}}=$ light off). This characteristic OR function is clearly demonstrated in Fig. 4(b). The output current modulation AIP license or copyright; see http://apl.aip.org/apl/copyright.jsp 

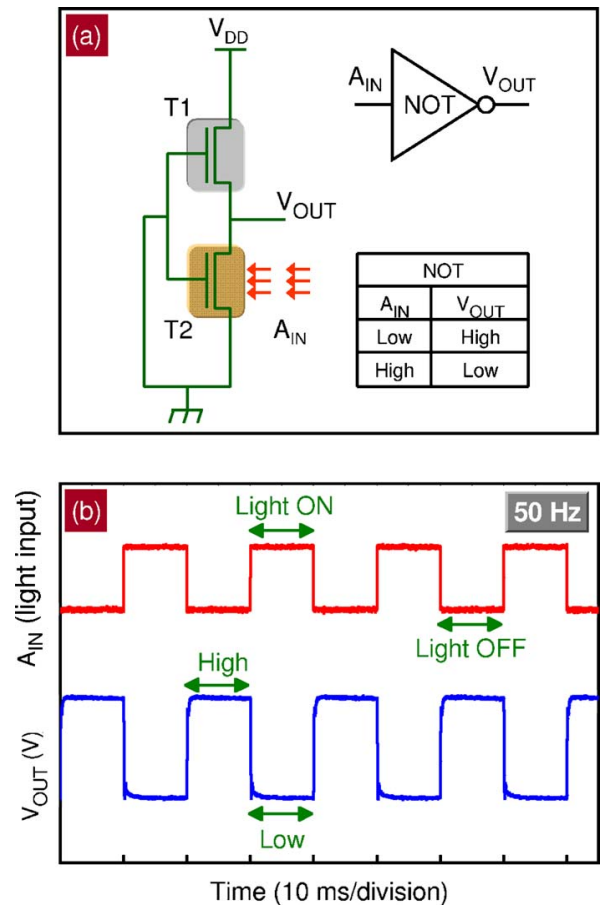

FIG. 3. (Color online) (a) Circuitry of the electro-optical NOT gate together with its symbolic representation and the truth table. (b) Optical input $A_{\mathrm{IN}}$ pulsed at $50 \mathrm{~Hz}$ (red trace) and the evolution of the output voltage $V_{\text {OUT }}$ (blue trace) in time.

ratio (i.e., $I_{\mathrm{OUT}(\text { light } \mathrm{ON})} / I_{\mathrm{OUT}}$ (light $\mathrm{OFF}$ ) is reasonably high and on the order of $10^{2}$ for both optical and electrical input signals. The latter, however, could be improved through appropriate transistor/circuit design. It should also be noted that a similar OR gate functionality could easily be achieved employing alternative circuit designs, however, at the expense of the circuits' complexity.

Finally, one could also envision fabrication of circuits where the optical input(s) are not only restricted to binary states of high and low but they may also contain analog/ continuous information. For example, the circuit of Fig. 3(a) can be designed so that $V_{\text {OUT }}$ is proportional to the intensity of $A_{\mathrm{IN}}$. This means that the analog information contained in the amplitude of $A_{\mathrm{IN}}$ may also be converted into electrical signals at the output. By going a step further and integrating wavelength-selective LS-OFETs (realized using either external optical filters or suitable semiconductor blends), fabrication of circuits with multiwavelength detection capabilities should be, in principle, possible. Such devices could find applications in full-color image sensor arrays. In this context, LS-OFETs offer the unique and interesting possibility of being integrated with the driving electronics (i.e., unipolar OFETs) as part of the same fabrication process without the need of additional processing steps typically required in photodiode-based organic sensors. ${ }^{10}$

In conclusion, electro-optical logic circuits based on light-sensing ambipolar OFETs with fast operating characteristics have been demonstrated. This is the only demonstration of organic circuits where signal processing involves the use of both optical and electrical input signals. Such optoelectronic circuits could one day be explored in various applications including electro-optical transceivers and optical sensor arrays.
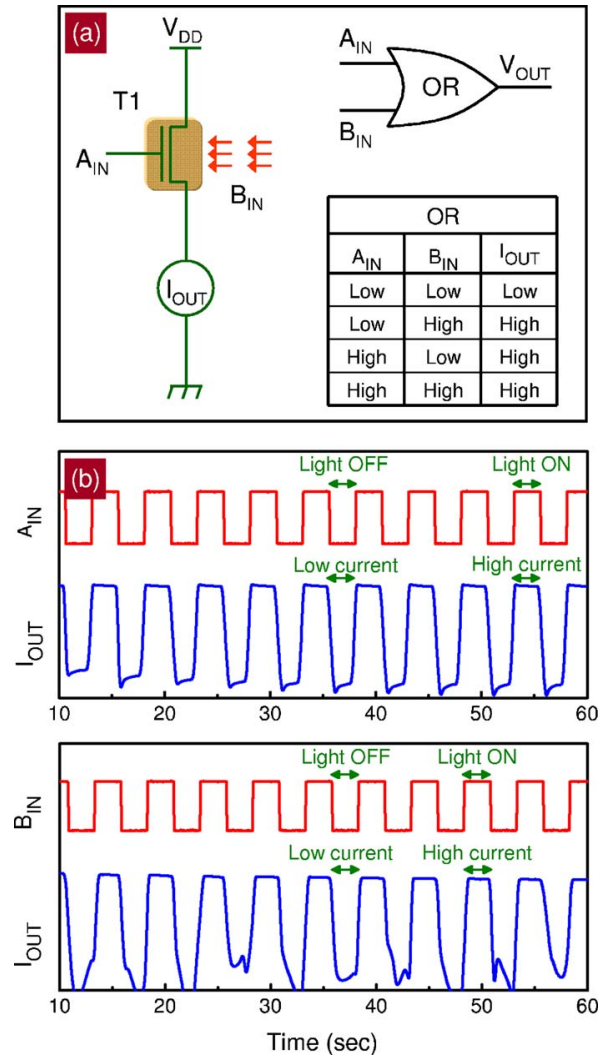

FIG. 4. (Color online) (a) Circuitry of the electro-optical OR gate together with its symbolic representation and the truth table. The circuit combines two inputs, one electrical $\left(A_{\mathrm{IN}}\right)$ and one optical $\left(B_{\mathrm{IN}}\right)$. (b) $I_{\mathrm{OUT}}, A_{\mathrm{IN}}$, and $B_{\mathrm{IN}}$ versus time.

The author is grateful to D. de Leeuw and D. Bradley for fruitful discussions and the Engineering and Sciences Research Council (EPSRC) for financial support.

${ }^{1}$ G. H. Gelinck, H. E. A. Huitema, E. van Veenendaal, E. Cantatore, L. Schrijnemakers, J. B. P. H. van der Putten, T. C. T. Geuns, M. Beenhakkers, J. B. Giesbers, B. -H. Huisman, E. J. Meijer, E. M. Benito, F. J. Touwslager, A. W. Marsman, B. J. E. van Rens, and D. M. de Leeuw, Nat. Mater. 3, 106 (2004)

${ }^{2}$ C. J. Drury, C. M. Mutsaers, C. M. Hart, M. Matters, and D. M. de Leeuw, Appl. Phys. Lett. 73, 108 (1998).

${ }^{3}$ B. Crone, A. Dodabalapur, Y.-Y. Lin, R. W. Fillas, Z. Bao, A. LaDuca, R. Sarpeshkar, H. E. Katz, and W. Li, Nature (London) 403, 521 (2000).

${ }^{4}$ A. Hepp, H. Heil, W. Weise, M. Ahles, R. Schmechel, and H. von Seggern, Phys. Rev. Lett. 91, 157406 (2003).

${ }^{5}$ J. S. Swensen, C. Soci, and Alan J. Heeger, Appl. Phys. Lett. 87, 253511 (2005).

${ }^{6}$ J. Zaumzeil, R. H. Friend, and H. Sirringhaus, Nat. Mater. 5, 69 (2006).

${ }^{7}$ N. Marjanovic, Th. B. Singha, G. Dennler, S. Gunes, H. Neugebauer, N.

S. Sariciftci, R. Schwodiauer, and S. Bauer, Org. Electron. 7, 188 (2006).

${ }^{8}$ Y.-Y. Noh, Dong-Yu Kim, and K. Yase, J. Appl. Phys. 98, 074505 (2005).

${ }^{9}$ S. Cho, J. Yuen, J.-Y. Kim, K. Lee, and A. J. Heeger, Appl. Phys. Lett. 90, 063511 (2007).

${ }^{10}$ T. Someya, Y. Kato, S. Iba, Y. Noguchi, T. Sekitani, H. Kawaguchi, and T. Sakurai, IEEE Trans. Electron Devices 52, 2502 (2005).

${ }^{11}$ E. C. P. Smits, T. D. Anthopoulos, S. Setayesh, B. de Boer, E. van Veenendaal, R. Coehoorn, P. W. M. Blom, and D. M. de Leeuw, Phys. Rev. B 73, 205316 (2006).

${ }^{12}$ T. D. Anthopoulos, D. M. de Leeuw, E. Cantatore, S. Setayesh, E. J. Meijer, C. Tanase, J. C. Hummelen, and P. W. M. Blom, Appl. Phys. Lett. 85, 4205 (2004).

${ }^{13}$ T. D. Anthopoulos, C. Tanase, S. Setayesh, E. J. Meijer, J. C. Hummelen, P. W. M. Blom, and D. M. de Leeuw, Adv. Mater. (Weinheim, Ger.) 16, 2174 (2004).

${ }^{14}$ The off state impedance of transistors $\mathrm{T} 1$ and $\mathrm{T} 2$ was carefully matched by their physical size, i.e., channel length $(L)$ and width $(W)$. 